SMAD, Rev Eletrônica Saúde Mental Álcool Drog.

2021 abr.-jun.; $17(2): 44-53$

DOI: 10.11606/issn.1806-6976.smad.2021.171786

www.revistas.usp.br/smad/

Artigo Original

\title{
Depressão em pacientes atendidos em serviço de saúde mental: fatores associados e diagnósticos de enfermagem
}

\author{
Ricardo Otávio Maia Gusmão1 \\ (D) https://orcid.org/0000-0001-9941-1114 \\ Natália Hiany Fonseca Santos ${ }^{2}$ \\ (D) https://orcid.org/0000-0002-7922-7093 \\ Daniel Vinicius Alves Silva ${ }^{3}$ \\ (D) https://orcid.org/0000-0001-9280-9146 \\ Deborah Fernanda Nunes Moreira ${ }^{1}$ \\ (D) https://orcid.org/0000-0003-4960-0245 \\ Maria Aparecida Vieira ${ }^{1}$ \\ (D) https://orcid.org/0000-0002-7921-4049 \\ Diego Dias de Araújo ${ }^{1}$ \\ (D) https://orcid.org/0000-0002-8927-6163
}

\footnotetext{
Universidade Estadual de Montes Claros, Montes Claros, MG, Brasil.

2 Hospital João XXIII, Belo Horizonte, MG, Brasil

3 Hospital Nossa Senhora das Mercês, Santa Casa, Montes
} Claros, MG, Brasil.

\begin{abstract}
Objetivo: estimar a prevalência de depressão em pacientes atendidos em Centro de Atenção Psicossocial, identificar os fatores sociodemográficos e diagnósticos de Enfermagem associados ao desfecho. Método: estudo transversal, realizado entre janeiro a junho de 2018, em 370 prontuários de pacientes atendidos, no período de 2002 a 2016, em Centro de Atenção Psicossocial de um município do norte de Minas Gerais, Brasil. Com o auxílio de um instrumento, foram coletados dados clínicos, sociodemográficos e diagnósticos de Enfermagem. Os diagnósticos de Enfermagem foram validados por especialistas com suporte no raciocínio diagnóstico de Risner. Realizaram-se análises descritiva, bivariada e regressão logística. Resultados: dos 370 pacientes, 57 (15,4\%) apresentaram depressão. A maioria $(53,2 \%)$ era composta por indivíduos do sexo feminino e a média de idade foi de 36 anos. Os diagnósticos de Enfermagem prioritários entre os pacientes com depressão foram: humor deprimido (100\%), crise $(68,4 \%)$, insônia $(45,6 \%)$ e risco de suicídio (49,1\%). As variáveis independentes que impactaram, de forma significativa, o desfecho depressão foram: sexo feminino $(p=0,012)$, insônia $(p=0,006)$ e risco de suicídio $(p<0,001)$. Conclusão: identificar precocemente possíveis fatores e diagnósticos de Enfermagem associados à pessoa com depressão poderá auxiliar na implementação de cuidados acurados a pacientes com esse problema atendidos em Centro de Atenção Psicossocial.
\end{abstract}

Descritores: Serviços de Saúde Mental; Depressão; Prevalência; Enfermagem; Diagnóstico de Enfermagem.

\section{Como citar este artigo}

Gusmão ROM, Santos NHF, Silva DVA, Moreira DFN, Vieira MA, Araújo DD. Depression in patients treated in a mental health service: prevalence and associated factors. SMAD, Rev Eletrônica Saúde Mental Álcool Drog. 2021 abr.-jun.;17(2):44-53. doi: https://dx.doi.org/10.11606/issn.1806-6976.smad.2021.171786 


\section{Depression in patients served in mental health service: prevalence and associated factors}

Objective: estimate the prevalence of depression in patients seen in a psychosocial care center, identify the sociodemographic factors and nursing diagnoses associated with the outcome. Method: cross-sectional study, carried out between January and June 2018, in 370 medical records of patients treated, from 2002 to 2016, in a psychosocial care center in a municipality in the north of Minas Gerais, Brazil. With the aid of an instrument, clinical, sociodemographic and nursing diagnoses data were collected. Nursing diagnoses were validated by specialists with support for Risner's diagnostic reasoning. Descriptive, bivariate analysis and logistic regression were performed. Results: of the 370 patients, 57 (15.4\%) had depression. The majority (53.2\%) were female and the average age was 36 years. The priority nursing diagnoses among patients with depression were: depressed mood $(100 \%)$, crisis $(68,4 \%)$, insomnia $(45,6 \%)$ and risk of suicide $(49,1 \%)$. The independent variables that significantly impacted the depression outcome were: female gender $(p=0,012)$, insomnia $(p=0,006)$ and risk of suicide $(p<0,001)$. Conclusion: early identification of possible nursing factors and diagnoses associated with a person with depression may help in the implementation of accurate care for patients with this problem treated at a psychosocial care center.

Descriptors: Mental Health Services; Depression; Prevalence; Nursing; Nursing Diagnosis.

\section{Depresión en pacientes atendidos en servicio de salud mental: prevalencia y factores associados}

Objetivo: estimar la prevalencia de depresión en pacientes atendidos en un centro de atención psicosocial, identificar los factores sociodemográficos y diagnósticos de enfermería asociados al resultado. Método: estudio transversal, realizado entre enero y junio de 2018, en 370 historias clínicas de pacientes atendidos, de 2002 a 2016, en un centro de atención psicosocial de un municipio del norte de Minas Gerais, Brasil. Con la ayuda de un instrumento se recolectaron datos de diagnósticos clínicos, sociodemográficos y de enfermería. Los diagnósticos de enfermería fueron validados por especialistas con apoyo del razonamiento diagnóstico de Risner. Se realizó análisis descriptivo, bivariado y regresión logística. Resultados: de los 370 pacientes, 57 (15,4\%) tenían depresión. La mayoría (53,2\%) eran mujeres y la edad media era de 36 años. Los diagnósticos de enfermería prioritarios entre los pacientes con depresión fueron: estado de ánimo deprimido (100\%), crisis $(68,4 \%)$, insomnio $(45,6 \%)$ y riesgo de suicidio $(49,1 \%)$. Las variables independientes que impactaron significativamente el resultado de la depresión fueron: sexo femenino $(p=0,012)$, insomnio $(p=0,006)$ y riesgo de suicidio $(p<0,001)$. Conclusión: la identificación temprana de posibles factores de enfermería y diagnósticos asociados a una persona con depresión puede ayudar en la implementación de una atención adecuada a los pacientes con este problema atendidos en un centro de atención psicosocial.

Descriptores: Servicios de Salud Mental; Depresión; Prevalencia; Enfermería; Diagnóstico de Enfermería. 


\section{Introdução}

Os transtornos mentais são definidos como quadros clínicos com manifestações psicológicas associadas ao comprometimento funcional devido a perturbações biológicas, sociais, psicológicas, genéticas, físicas ou químicas. Podem ocasionar alterações no desempenho global do indivíduo nos âmbitos pessoal, social, ocupacional ou familiar(1).

Dentre os transtornos mentais, a depressão pode ser classificada como um dos principais e mais recorrentes problemas de saúde mental, com prevalência de até $20 \%$ na população mundial. Impacta o meio social de tal modo que é reconhecida como o segundo quadro clínico a produzir mais danos nas esferas social e econômica(2).

Os transtornos depressivos são descritos segundo a Classificação Internacional de Doenças (CID 11), da Organização Mundial de Saúde (OMS). Sua sintomatologia tem como base central as alterações do humor e afeto. A tristeza apresenta-se como sintoma nuclear, no entanto, além de alterações do humor, como a hipotimia, a apatia, a anedonia e a irritabilidade, podem ocorrer também alterações cognitivas, motoras e vegetativas, com destaque para as alterações de sono e apetite ${ }^{(3)}$.

Os sintomas depressivos podem estar presentes em outros quadros clínicos, tais como a esquizofrenia, o alcoolismo, a demência, doenças clínicas ou mesmo situações específicas, como o luto e o estresse. Neste sentido, esses sintomas, quando presentes, mesmo não configurando o diagnóstico de algum tipo de depressão, são capazes de produzir dificuldades no desempenho do trabalho e prejuízos sociais(4).

Nessa conjuntura, o cuidado com indivíduos depressivos deve ser realizado por uma equipe multidisciplinar que trabalha de forma interdisciplinar. Dentre os membros da equipe, o enfermeiro deve atuar na identificação de necessidades e na implementação de cuidados de Enfermagem a esses pacientes. Sua prática clínica tem como base a escuta, o diálogo, o afeto, o acolhimento, o conforto e o foco no relacionamento terapêutico centrado na pessoa(5).

A ampliação da clínica no campo da Saúde Mental iniciou-se no Brasil no final da década de 1970, marcada pela Reforma Psiquiátrica Brasileira (RPB), que se caracterizou pela reestruturação do modelo assistencial com a implantação da Estratégia de Atenção Psicossocial (EAPS). A EAPS foi expandida por meio do desenvolvimento da Rede de Atenção Psicossocial (RAPS)(6).

Desse modo, o Brasil garantiu um panorama favorável ao atendimento aos quadros depressivos, pois possibilitou mais acesso ao tratamento do usuário com depressão(2). Diante dessa realidade, o cuidado de Enfermagem em Saúde Mental deve, então, promover o suporte psicossocial, o conforto, além dos cuidados necessários a este contexto. Para isso, é necessário aplicar a Sistematização da
Assistência de Enfermagem (SAE), preconizando a implementação do Processo de Enfermagem $(P E)^{(7)}$.

Há carência na literatura de estudos que tenham gerado informações sobre a prevalência, fatores sociodemográficos e diagnósticos de Enfermagem associados à depressão em pacientes atendidos em Centro de Atenção Psicossocial (CAPS). Diante da importância desse tema para a área da saúde e Enfermagem e da vulnerabilidade da população ao problema, percebe-se a necessidade de verificar a ocorrência, bem como os possíveis fatores e diagnósticos de Enfermagem associados ao desfecho neste perfil de pacientes. Assim, este estudo teve como objetivo estimar a prevalência de depressão em pacientes atendidos em CAPS, identificar os fatores sociodemográficos e os diagnósticos de Enfermagem associados ao desfecho.

\section{Método}

Trata-se de um estudo transversal, realizado de janeiro a junho de 2018 , em prontuários de pacientes atendidos, no período de 2002 a 2016, no CAPS de um município do norte de Minas Gerais, Brasil. Destaca-se que a instituição não utiliza linguagem padronizada de diagnósticos de Enfermagem.

A estrutura física do CAPS em questão comporta até 30 pacientes e realiza de 30 a 40 atendimentos por dia. 0 local é composto por dois psiquiatras, dois enfermeiros, cinco psicólogos, seis técnicos de Enfermagem, um farmacêutico e uma monitora de oficina.

Na RAPS, o CAPS tem como propósito atender o paciente em crise originário do município de estudo a partir dos três níveis de atendimentos: intensivo, semi-intensivo e não intensivo. O paciente pode vir encaminhado da ESF ou de alguma unidade da rede municipal de saúde; do Serviço de Atendimento Móvel de Urgência (SAMU); do Corpo de Bombeiros ou da Polícia Militar ou por demanda espontânea.

Entre 2002 e 2016, foi admitido, no CAPS do município do estudo, um total de 1918 pacientes. Foi determinada uma amostra, por meio de cálculo probabilístico e estratificado, resultando em, no mínimo, 370 prontuários. Com isso, foram realizados cálculos para determinar o número de prontuários por ano que seriam pesquisados, a saber: $2002-21 ; 2003-49 ; 2004-30$; 2005 - 22; 2006 - 9; 2007 - 16; 2008 - 18; 2009 - 27; 2010 - 23; 2011 - 18; 2012 - 16; 2013 - 32; 2014 - 26; 2015 - 35; 2016 - 29, totalizando 370 pacientes. Os prontuários encontram-se na forma impressa no CAPS do município e foram selecionados por sorteio simples.

O procedimento de coleta de dados foi realizado por uma graduanda, do sétimo período do curso de graduação em Enfermagem de uma universidade pública do Estado de Minas Gerais, devidamente capacitada e sob supervisão do professor orientador. 
Para nortear a coleta de dados, foi utilizado um instrumento, elaborado pelos pesquisadores, com as seguintes variáveis sociodemográficas: idade; sexo; estado civil; escolaridade; ocupação; presença de filhos; cuidador. As variáveis relacionadas ao perfil clínico foram: início do tratamento; origem do encaminhamento; tipo de internação; internação anterior; diagnóstico ou hipótese diagnóstica apresentada de acordo com a CID-10; classe de medicamentos; comorbidades clínicas; tabagismo; etilismo; drogas ilícitas.

Destaca-se que, anterior à coleta de dados, foi realizado um pré-teste para se verificar falhas que pudessem interferir negativamente na coleta de dados e nos resultados finais do estudo. Para tanto, foram escolhidos, aleatoriamente, documentos de pacientes fora do período em que foi realizado o estudo e esses não fizeram parte da pesquisa final.

Para a identificação dos diagnósticos de Enfermagem prioritários na admissão no CAPS, foram analisadas as fichas de admissão dos pacientes no serviço. Identificaram-se os termos ou as expressões em campos livres das respostas humanas em nível de bem-estar ou que necessitariam de intervenções específicas de Enfermagem. Estes foram a base para a identificação dos diagnósticos de Enfermagem constantes entre os termos/ conceitos pré-coordenados da CIPE ${ }^{\circledR}$, versão $2017^{(8)}$.

Para a validação dos achados, utilizou-se a técnica de validação por consenso(9-11), que propõe a análise por um grupo de enfermeiros clínicos, mínimo de três e máximo de cinco, com o intuito de estabelecer a opinião consensual $(100 \%)$ de especialistas sobre a pertinência e a relevância de determinado termo/expressão ou diagnóstico de Enfermagem. A discordância de um ou mais especialistas condicionou a não validação dos respectivos termos/ expressões ou diagnósticos de Enfermagem ${ }^{(10-11)}$. Os critérios de inclusão dos especialistas foram: ser enfermeiro(a); ter atuação profissional/residência em saúde, com duração mínima de dois anos de prática clínica de assistência em saúde mental; autor(a), coautor(a) ou orientador(a) de estudos envolvendo saúde mental. Selecionaram-se três especialistas, considerando a proximidade geográfica para a realização dos encontros presenciais.

O processo de validação por consenso ocorreu em um município do norte de Minas Gerais, em que quatro especialistas participaram de três encontros presenciais e, norteados pelas habilidades de sistematização do raciocínio diagnóstico de Risner ${ }^{(12-13)}$, validaram os diagnósticos de Enfermagem.

Com base no fundamento de raciocínio de Risner(12-13), aplicou-se o raciocínio analítico e sintético, atentando às habilidades de pensamento crítico, raciocínio clínico ${ }^{(14)}$, o conhecimento científico e experiências dos enfermeiros em correspondência às inferências elaboradas a partir dos elementos presentes nas fichas de admissão do paciente no CAPS e os termos/conceitos pré-coordenados da CIPE ${ }^{\circledR}$, versão $2017^{(8)}$.

Após a coleta, os dados foram inseridos em uma planilha eletrônica do Microsoft Excel 2013 e exportados para o Statistical Package for Social Science (SPSS), versão 20.0. Foi conduzida a análise descritiva (frequências simples e percentual). A prevalência foi estabelecida considerando o número de pacientes (casos novos e antigos) que relataram o desfecho durante o período de coleta de dados.

Empregou-se análise bivariada para as variáveis estudadas a partir dos testes de qui-quadrado e exato de Fisher. Com isso, obteve-se a relação entre cada variável independente e a variável desfecho (depressão), sendo medida a força de associação pelo Odds Ratio (OR), considerando o Intervalo de Confiança (IC) de $95 \%$. Para a identificação das covariáveis pesquisadas que exerceram influência sobre o desfecho, utilizou-se o modelo de regressão logística, sendo medida a força de associação pelo OR. Variáveis cujo valor de $p$ foi $\leq 0,20$ na análise bivariada foram incluídas no modelo de análise multivariada. Para a análise final, considerou-se um nível de significância final de 0,05 $(p<0,05)$.

O estudo obteve parecer favorável do Comitê de Ética em Pesquisa da Universidade Estadual de Montes Claros (Unimontes) sob o parecer de CAAE: 71723417.5.0000.5146.

\section{Resultados}

Do total de 370 pacientes atendidos no CAPS, a prevalência de depressão foi de $15,4 \%(n=57)$ (Tabela 1).

Tabela 1 - Características clínicas dos pacientes atendidos no CAPS*. Montes Claros, MG, Brasil, 2016-2017

\begin{tabular}{lcc}
\hline Variáveis & $\mathbf{n}$ & $\%$ \\
\hline Transtornos Mentais & 187 & 50,5 \\
Esquizofrenia & 57 & 15,4 \\
Depressão & 55 & 14,9 \\
Transtornos Neuróticos Estresse e & & \\
Somatoformes & 41 & 11,1 \\
Transtornos Esquizotímicos e & 40 & 10,8 \\
Transtornos Delirantes & 20 & 5,4 \\
Transtorno afetivo bipolar & 19 & 5,1 \\
Retardo mental & & \\
Transtornos Mentais e & &
\end{tabular}

Fonte: cenário do estudo, 2016-2017. ${ }^{*}$ CAPS = Centro de Atenção Psicossocial

Entre os 370 pacientes, destacam-se o sexo feminino $(n=197 ; 53,2 \%)$; solteiros $(n=218 ; 58,9 \%)$; com idades entre 18 e 76 anos e média de 36 anos. Quanto à escolaridade, observou-se que a maioria cursou o Ensino Fundamental incompleto $(n=153 ; 41,4 \%)$; não trabalhava $(n=283 ; 76,5 \%)$; não tinha filhos $(n=211 ; 57 \%)$ e 300 $(81,1 \%)$ possuíam cuidador/acompanhante (Tabela 2 ). 
Tabela 2 - Variáveis sociodemográficas dos pacientes atendidos em um CAPS*. Montes Claros, MG, Brasil, 2016-2017

\begin{tabular}{lcc}
\hline Variáveis & $\mathbf{n}$ & $\%$ \\
\hline Sexo & & \\
Masculino & 173 & 46,8 \\
Feminino & 197 & 53,2 \\
\hline Estado Civil & & \\
Solteiro & 218 & 58,9 \\
Casado & 109 & 29,5 \\
Divorciado & 27 & 7,3 \\
Viúvo & 11 & 3,0 \\
Sem informação & 5 & 1,4 \\
\hline Escolaridade & & \\
Analfabeto & 26 & 7,0 \\
Ensino Fundamental incompleto & 153 & 41,4 \\
Ensino Fundamental completo & 30 & 8,1 \\
Ensino Médio incompleto & 36 & 9,7 \\
Ensino Médio completo & 74 & 20,0 \\
Superior & 16 & 4,3 \\
Sem informação & 35 & 9,5 \\
\hline Ocupação & & \\
Não trabalha & 283 & 76,5 \\
Trabalha & 71 & 19,2 \\
Estudante & 12 & 3,2 \\
Sem informação & 4 & 1,1 \\
\hline Filhos & & \\
Não & 211 & 57,0 \\
Sim & 153 & 41,4 \\
Sem informação & 6 & 1,6 \\
\hline Cuidador/Acompanhante & & \\
Não & 70 & 81,1 \\
Sim & 300 & \\
\hline & & \\
\hline
\end{tabular}

Fonte: cenário do estudo, 2016-2017. *CAPS = Centro de Atenção Psicossocial
Identificaram-se, entre os pacientes do CAPS, 54 $(14,5 \%)$ hipertensos; $67(18,1 \%)$ tabagistas e $53(14,3 \%)$ etilistas.

Os diagnósticos de Enfermagem prioritários na admissão no CAPS foram: "crise" $(n=298 ; 80,5 \%)$; "comportamento agressivo" ( $n=128 ; 34,6 \%)$; "delírio" ( $n=125 ; 33,8 \%)$; "alucinação" ( $n=121 ; 30,8 \%)$; "insônia" ( $n=113 ; 30,5 \%)$; "agitação" $(n=106 ; 28,6 \%)$; "humor deprimido" ( $n=96 ; 25,9 \%)$ e "risco de suicídio" ( $n=49$; $13,2 \%$ ). Já os DE prioritários entre os pacientes com depressão foram: "humor deprimido" $(n=57 ; 100 \%)$; "crise" ( $n=39,68,4 \%)$; "insônia" ( $n=26,45,6 \%)$ e "risco de suicídio" ( $n=28,49,1 \%$ ).

A Tabela 3 apresenta as variáveis que apresentaram significância estatística com a depressão por meio da análise bivariada.

Tabela 3 - Associação entre a variável dependente, depressão, e variáveis independentes em pacientes atendidos em um CAPS*. Montes Claros, MG, Brasil, 2016-2017

\begin{tabular}{|c|c|c|c|c|c|c|c|}
\hline \multirow{3}{*}{ Características } & \multirow{3}{*}{ Grupo } & \multicolumn{4}{|c|}{ Depressão } & \multirow{3}{*}{$\mathrm{OR}^{\dagger}(\mathrm{IC} 95 \%)$} & \multirow{3}{*}{ P-valor } \\
\hline & & \multicolumn{2}{|c|}{ Sim } & \multicolumn{2}{|c|}{ Não } & & \\
\hline & & $\mathbf{N}$ & $\%$ & $\mathbf{N}$ & $\%$ & & \\
\hline \multirow{3}{*}{ Sexo } & Feminino & 42 & 21,3 & 155 & 78,7 & \multirow{3}{*}{$2,854(1,520-5,358)$} & \multirow{3}{*}{0,001} \\
\hline & & & & & & & \\
\hline & Masculino & 15 & 8,7 & 158 & 91,3 & & \\
\hline \multirow{3}{*}{ Escolaridade } & Analfabeto & 3 & 11,5 & 23 & 88,5 & \multirow{3}{*}{$0,676(0,195-2,336)$} & \multirow{3}{*}{0,533} \\
\hline & & & & & & & \\
\hline & Alfabetizado & 50 & 16,2 & 259 & 83,8 & & \\
\hline \multirow{3}{*}{ Insônia } & Sim & 26 & 23,0 & 87 & 77,0 & \multirow{3}{*}{$2,179(1,224-3,879)$} & \multirow{3}{*}{0,007} \\
\hline & & & & & & & \\
\hline & Não & 31 & 12,1 & 226 & 87,9 & & \\
\hline \multirow{3}{*}{ Risco de Suicídio } & Sim & 28 & 57,1 & 21 & 42,9 & \multirow{3}{*}{$13,425(6,785-26,565)$} & \multirow{3}{*}{$<0,001$} \\
\hline & & & & & & & \\
\hline & Não & 29 & 9,0 & 292 & 91,0 & & \\
\hline \multirow{3}{*}{ Ansiedade } & Sim & 8 & 30,8 & 18 & 69,2 & \multirow{3}{*}{$2,160(1,148-4,065)$} & \multirow{3}{*}{$0,042^{\ddagger}$} \\
\hline & & & & & & & \\
\hline & Não & 18 & 25,0 & 313 & 84,6 & & \\
\hline \multirow{3}{*}{ Humor Deprimido } & Sim & 57 & 59,4 & 39 & 40,6 & \multirow{3}{*}{-} & \multirow{3}{*}{$<0,001$} \\
\hline & & & & & & & \\
\hline & Não & 0 & 0 & 274 & 100 & & \\
\hline Idade & - & \multicolumn{4}{|c|}{$37,12^{\S} \pm 12,52^{\|}$} & - & 0,265 \\
\hline
\end{tabular}

Fonte: cenário do estudo, 2016-2017. *CAPS = Centro de Atenção Psicossocial; ${ }^{\dagger}$ OR = Odds Ratio; ${ }^{\ddagger}$ Teste de Fisher; ${ }^{\S}$ Média; $\mid$ Desvio-Padrão 
As características sociodemográficas e os diagnósticos de Enfermagem prioritários identificados nos pacientes - sexo (feminino), "insônia" e "risco de suicídio" impactaram, de forma significativa, a depressão na análise multivariada (Tabela 4).

Tabela 4 - Fatores associados à depressão em pacientes atendidos por um CAPS*. Montes Claros, MG, Brasil, 2016-2017

\begin{tabular}{lcccc}
\hline \multirow{2}{*}{ Variável Independente } & \multirow{O}{*}{$\boldsymbol{R}^{\dagger}$} & \multicolumn{1}{l}{ IC+95\% } & OR & \multirow{2}{*}{ P-valor } \\
\cline { 3 - 4 } & & Inferior & Superior & \\
\hline Sexo (Feminino) & 2,442 & 1,215 & 4,909 & 0,012 \\
Insônia & 2,539 & 1,302 & 4,954 & 0,006 \\
Risco de Suicídio & 13,407 & 6,545 & 27,461 & $<0,001$ \\
\hline
\end{tabular}

Fonte: cenário do estudo, 2016-2017. ${ }^{*}$ CAPS = Centro de Atenção Psicossocial; ${ }^{\dagger} \mathrm{IC}=$ Intervalo de Confiança; ${ }^{\ddagger} \mathrm{OR}=$ Odds Ratio

\section{Discussão}

Neste estudo, a prevalência de depressão em pacientes atendidos por um CAPS foi de 15,4\%, aproximada à encontrada em estudos prévios, que apresentaram prevalência entre $14,4 \%$ e $19,5 \%{ }^{(15-17)}$.

Segundo a OMS, cerca de $4,4 \%$ da população mundial possui depressão. No Brasil, essa prevalência é maior, 5,8\%(4). Ainda segundo relatório da OMS, houve o significativo aumento de $18 \%$ dos casos de depressão entre 2005 e 2015, sendo a maioria em mulheres ${ }^{(18)}$.

Os transtornos depressivos configuram-se como problema de saúde pública na contemporaneidade em decorrência de sua alta prevalência, impactos psicossociais produzidos e repercussões na saúde de modo geral. Para - Diagnostic and Statistical Manual of Mental Disorders (DSM-V), devem ser considerados, para o diagnóstico de depressão, os seguintes critérios: hipotimia (estado deprimido em maior parte do tempo); anedonia (perda de prazer ou desinteresse para a realização de atividades cotidianas); sentimento de inutilidade ou culpa excessiva; fadiga; perda de concentração; distúrbios do sono; alterações psicomotoras, ganho ou perda significativa de peso; ideias de morte ou suicídio(19).

A casuística da depressão não pode ser atribuída a apenas um fator. Assim, como a maioria dos problemas humanos, é recomendável ampliar sua análise e considerar múltiplos fatores que interagem, produzindo respostas e comportamentos que os sujeitos apresentam em seu contexto social(20).

Atualmente, no Brasil, o cuidado em saúde mental deve ser orientado pelo modelo de rede, buscando-se ações interdisciplinares e intersetoriais. Essas ações devem fundamentar-se nos princípios da Reforma Psiquiátrica (RP) e do Sistema Único de Saúde (SUS), buscando a ampliação da clínica com resgate e valorização das dimensões psicossociais.
O sujeito em sofrimento psíquico deve ser cuidado, visando à sua reabilitação psicossocial, desinstitucionalização e reinserção social. Nesta perspectiva, o CAPS emerge como dispositivo importante no cuidado de indivíduos depressivos. Funciona como serviço intermediário entre o regime ambulatorial e a internação hospitalar, sendo composto por equipes multiprofissionais. Deve ser um serviço de referência para situações de crise e de grande vulnerabilidade clínica e social, principalmente situações que envolvam risco de morte. Seu foco é o acompanhamento de usuários com transtornos mentais graves e persistentes de um território específico, em regime de tratamento intensivo, semi-intensivo e não intensivo ${ }^{(21)}$.

Entre os indivíduos deste estudo, a idade variou entre 18 e 76 anos, com média de 36 anos, 58,9\% eram solteiros e $41,4 \%$ possuíam baixa escolaridade, com Ensino Fundamental incompleto. É possível observar que, no CAPS em questão, a maioria dos usuários com transtornos mentais está na faixa etária produtiva ou economicamente ativa, porém, 76,5\% dos pacientes não trabalham.

Esses dados são corroborados por outros estudos ${ }^{(15-17)}$ conduzidos em CAPS de regiões distintas do Brasil. No estudo conduzido em Passos (MG), a idade variou entre 18 e 79 anos, sendo a maioria dos indivíduos solteira (46,5\%) e com Ensino Fundamental incompleto (59\%) (16). Em Curitiba (PR), 44,7\% eram solteiros e 38,4\%,

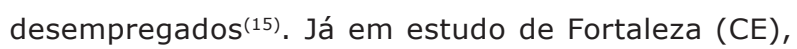
a média de idade foi de 46 anos, com predomínio de pacientes com quatro a sete anos de estudo $(9,6 \%)$ e $62 \%$ encontravam-se sem atividade laborativa formal ${ }^{(17)}$.

Destaca-se que os sujeitos em sofrimento psíquico ainda vivenciam muitos estigmas sociais, tais como o abandono dos estudos, muitas vezes, devido às limitações impostas pelos transtornos mentais e o demérito de que estes são desqualificados e incapazes de manter relações trabalhistas. Entretanto, estas são questões importantes e modificáveis por estratégias inclusivas no setor da educação e do trabalho. A educação, a possibilidade de qualificação e a inserção no mercado de trabalho são relevantes no processo de reabilitação psicossocial, autonomia e cidadania(15,22-23).

Dos 370 pacientes, 300 (81,1\%) tinham acompanhantes ou cuidadores durante o tratamento no CAPS. Com a RPB e a reestruturação do modelo assistencial, ocorreu gradativamente a desinstitucionalização, assim, principalmente a família passou a ser a protagonista no processo de cuidado.

Neste estudo, houve significância estatística entre depressão e sexo (feminino) (OR:2,442; $p=0,012)$, insônia (OR:2,539; $\mathrm{p}=0,006)$ e risco de suicídio (OR:13,407; $\mathrm{p}<0,001)$.

Em relação à depressão e ao sexo, neste estudo, $21,3 \%$ dos pacientes diagnosticados com o desfecho 
eram do sexo feminino. Esse dado é reforçado por outros estudos ${ }^{(17,24)}$.

Em metanálise ${ }^{(25)}$ de estudos de depressão em adultos brasileiros, encontrou-se uma prevalência do problema em 21,6\% (IC 95\%: 18,5-24,7) das mulheres e $9,7 \%$ (IC 95\%: 7,5-11,8) dos homens. Em outro estudo de base populacional, que incluiu 1.593 adultos do Distrito Federal, a depressão foi autorreferida em 14,6\% (IC 95\%: $12,0-17,6 \%$ ) entre as mulheres ${ }^{(26)}$.

A associação entre a depressão e o sexo feminino é apontada(27) como sendo atribuída a diversos fatores como: causas hormonais; gestação; puerpério e maior busca pelos serviços de saúde. Autores explicam que as mulheres teriam maior facilidade de identificar seu sofrimento psíquico, admiti-lo e buscar ajuda, enquanto os homens tendem a usar substâncias psicoativas como alívio para seu sofrimento ou angústia(27).

Estudos $^{(2,26,28)}$ têm sustentado que a depressão seja aproximadamente duas vezes mais comum em mulheres do que em homens. Além dos fatores supracitados, essa ocorrência também tem sido explicada pelas diferenças hormonais e fisiológicas, baixo nível de escolaridade, questões socioculturais, baixa renda e particularidades nas formas de vivenciar situações estressoras na vida(25-26,28).

Na perspectiva do cuidado, destaca-se a Enfermagem, profissão comprometida com o ato de cuidar em todas as fases do desenvolvimento humano, inclusive durante a assistência ao paciente em sofrimento psíquico.

Uma forma de se propiciar, ao paciente em sofrimento psíquico, um cuidado de Enfermagem de excelência e ordenado é por meio da SAE e pela implementação do PE. Trata-se do método clínico da profissão que guia o cuidado de Enfermagem, sendo composto por cinco etapas: coleta de dados; diagnóstico de Enfermagem; planejamento; implementação e avaliação de Enfermagem(29). Taxonomias de Enfermagem e linguagens padronizadas podem ser utilizadas para se determinar os diagnósticos de Enfermagem, as intervenções e os resultados de Enfermagem ${ }^{(30)}$.

Nesse sentido, a CIPE ${ }^{\circledR}$ é um sistema de classificação em Enfermagem que se encontra em vasto progresso global, com potencial aplicabilidade nos diversos níveis de atenção à saúde, contribuindo para a Enfermagem baseada em evidências científicas e para a confirmação da eficácia da assistência de Enfermagem por meio de indicadores da prática clínica, como é o caso dos diagnósticos de Enfermagem ${ }^{(8,31)}$.

O enunciado dos diagnósticos de Enfermagem refere-se a um título atribuído pelo enfermeiro a uma decisão sobre um fenômeno que é o foco da prática de Enfermagem ${ }^{(8)}$. Trata-se do julgamento clínico dos enfermeiros na identificação de problemas que são de competência da Enfermagem. Seu reconhecimento permite uma assistência holística, centrada no paciente, além de proporcionar o planejamento e a implementação de ações e intervenções de Enfermagem direcionadas para as reais necessidades do paciente.

Em estudo realizado em Portugal, em um serviço de urgência psiquiátrica, identificaram-se, com maior prevalência entre as internações, as perturbações depressivas. Verificou-se também que os diagnósticos de Enfermagem prioritários eram do domínio do humor, como "ansiedade", "tristeza", "humor depressivo" e "baixa autoestima"(32).

O diagnóstico de Enfermagem "insônia" ou "sono prejudicado", neste estudo, impactou, de forma significativa, a depressão. Esse distúrbio refere-se ao estado em que o indivíduo apresenta uma mudança na quantidade ou na qualidade do seu padrão de sono, causando desconforto ou interferindo no seu estilo de vida desejável. Essas perturbações do sono podem ser resultantes de fatores fisiológicos, psicológicos, sociais e ambientais, como estresse emocional, ansiedade, dor, desconforto, tensão, abuso de drogas ou distúrbio da função cerebral. A serotonina é um neurotransmissor com um papel fundamental na regulação do sono. $\mathrm{Na}$ depressão, ocorre uma diminuição funcional da serotonina, resultando em alterações específicas do sono(33-34).

O distúrbio do sono consiste em uma das principais alterações presentes do transtorno depressivo. Dos clientes com transtorno depressivo, cerca de $80 \%$ apresentam intercorrências na quantidade e qualidade do sono, com queixas frequentes de insônia, redução do sono total, despertares noturnos frequentes, sono não restaurador, além de sonhos perturbadores ${ }^{(35)}$.

Enfatiza-se que os transtornos do sono podem favorecer um novo episódio depressivo como ter sido o fator contribuinte para o surgimento da depressão. Outro dado importante refere-se à relação estabelecida entre pior qualidade do sono e distúrbios do sono com maior tendência a risco de suicídio(35-36).

O diagnóstico de Enfermagem "risco de suicídio" também se manteve, neste estudo, significativamente associado à depressão. As pessoas com transtorno mental têm maior risco de cometer suicídio quando comparadas às pessoas sem enfermidade psíquica. Estima-se que 95\% das pessoas que tentam ou cometem suicídio têm um transtorno mental previamente diagnosticado. Entre os transtornos mentais associados ao suicídio, a depressão maior destaca-se. A maioria dos indivíduos que tentam suicídio apresenta histórico de uma tentativa prévia, manifestando um risco maior de novas tentativas ${ }^{(15,36)}$.

A associação estabelecida entre a depressão maior e o suicídio está bem descrita pela literatura. Tal fenômeno confirmou-se em estudo epidemiológico do Canadá(37). A depressão é o principal quadro clínico que tem relação com a ideação suicida, tentativa de suicídio e planos suicidas. Em relação aos transtornos de ansiedade, a depressão 
maior esteve associada, em uma razão de chances, cerca de dez vezes mais $(\mathrm{OR}=1,9-18,5)^{(38)}$, o que se aproxima ao achado desta pesquisa $(\mathrm{OR}=13,407)$.

Na depressão, há de se considerar a existência de vulnerabilidades e fatores de risco que podem influenciar o cometimento do suicídio. Em deprimidos, há a redução dos níveis de serotonina, proporcionando, no cérebro, a impulsividade. Ao lidar com situações estressantes, esses indivíduos podem utilizar-se da agressividade contra si mesmo e a sua vida(36).

A OMS estimou que, a cada ano, um contingente de mais de 800 mil pessoas comete suicídio. O suicídio é a segunda causa mais frequente de morte entre pessoas de 19 a 25 anos de idade ${ }^{(39)}$.

Em 2004, no Estado de Minas Gerais, Brasil, a taxa de suicídio foi de 4,82 para cada 100 mil habitantes ${ }^{(40)}$.

É importante ressaltar que o humor deprimido ou a tristeza profunda fazem parte dos critérios para o diagnóstico de depressão, além da anedonia, inibição ou ausência da capacidade de sentir alegria ou prazer e a hipoatividade/fadiga, redução da energia e fatigabilidade aumentada(36).

A complexidade em lidar com a 'insônia" e o "risco de suicídio" produz diversos desafios para o cuidado clínico de Enfermagem em saúde mental. Nesse sentido, é fundamental o desenvolvimento de competências profissionais e pessoais para se implementar intervenções diante de situações de cunhos psíquico e emocional.

No que se refere à "insônia", o cuidado de Enfermagem pode ser implementado buscando-se destacar orientações sobre padrão de sono, obter dados sobre o sono e fatores que podem comprometê-lo, promover rotina de hora de dormir, avaliar resposta à medicação em caso de tratamentos psiquiátricos e orientar sobre dieta. Por sua vez, em relação ao "risco de suicídio", as principais intervenções devem possibilitar obter dados sobre adesão ao regime de segurança, sobre o humor deprimido, reforçar o controle de impulsos, facilitar o acesso ao tratamento e a capacidade para comunicar sentimentos, identificar percepções alteradas e monitorar adesão à medicação(8,31).

A identificação da prevalência de depressão, dos fatores sociodemográficos e dos diagnósticos de Enfermagem prioritários para a clientela específica, no caso, os pacientes com depressão atendidos em CAPS, colabora na descrição, comparação e representação do domínio da prática de Enfermagem para promover cuidados de qualidade baseados em evidências científicas. Tem-se, ainda, como contribuição no contexto da saúde e de Enfermagem, a aplicabilidade do PE na prática clínica do enfermeiro no CAPS.

Evidencia-se, como limitação do estudo, a carência de pesquisas que avaliem o desfecho depressão e a identificação de diagnósticos de Enfermagem CIPE $^{\circledR}$, especificamente, para pacientes atendidos em CAPS. Por ser um estudo transversal, não é possível investigar as condições de baixa prevalência, factível em desenhos longitudinais, identificar as relações de causa e efeito ou avaliar a evolução dos diagnósticos de Enfermagem no cuidado de Enfermagem no CAPS.

\section{Conclusão}

Este estudo possibilitou a identificação da prevalência de depressão em pacientes atendidos em CAPS do norte de Minas Gerais, bem como a identificação dos fatores sociodemográficos e diagnósticos de Enfermagem associados ao desfecho.

Após a análise bivariada e a etapa de ajuste da análise multivariada, dentre os fatores sociodemográficos e diagnósticos de Enfermagem, os que permaneceram como melhores preditores para o desfecho em estudo foram: "insônia", "risco de suicídio" e sexo (feminino).

$\mathrm{Na}$ prática clínica do enfermeiro, reconhecer precocemente os possíveis fatores e diagnósticos de Enfermagem associados à depressão pode auxiliá-lo e à equipe multiprofissional na gestão do cuidado, no planejamento e na implementação de intervenções direcionadas para as reais necessidades dos pacientes com depressão atendidos em CAPS.

Recomenda-se a realização de investigações que permitam explorar e estabelecer as diferenças relativas à depressão entre homens e mulheres, a relação entre fatores causais, diagnósticos de Enfermagem e os melhores cuidados de Enfermagem a serem implementados.

\section{Referências}

1. Quadros LCM, Quevedo LA, Gonçalves HD, Horta BL, Motta JVS, Gigante DP. Common Mental Disorders and Contemporary Factors: 1982 Birth Cohort. Rev Bras Enferm. 2020;73(1):e20180162. doi: http://doi. org/10.1590/0034-7167-2018-0162

2. Motta CCL, Moré CLOO, Nunes CHSS. Psychological assistance provided to patients diagnosed with depression in primary care. Cienc Saúde Coletiva. 2017;22(3):911-20. doi: http://doi.org/10.1590/1413-81232017223.27982015 3. Dalgalarrondo P. Psicopatologia e semiologia dos transtornos mentais. 3. ed. Porto Alegre: Artmed; 2018. $520 \mathrm{p}$

4. Facioli AM, Barros AB, Melo MC, Ogliari ICM, Custódio RJM. Depression among nursing students and its association with academic life. Rev Bras Enferm. 2020;73(1):e20180173. doi: http://doi.org/10.1590/0034-7167-2018-0173

5. Fontão MC, Rodrigues J, Lino MM, Lino MM, Kempfer SS. Nursing care to people admitted in emergency for attempted suicide. Rev Bras Enferm. 2018;71(suppl 5):2199-205. [Thematic Issue: Mental health]. doi: http:// doi.org/10.1590/0034-7167-2017-0219 
6. Silva PO, Silva DVA, Rodrigues CAO, Santos NHF, Barbosa SFA, Souto VD, et al. Nursing clinical care in mental health mental. Rev Enferm UFPE On Line. 2018;12(11):3133-46. doi: http://doi. org/10.5205/1981-8963-v12i11a236214p3133-3146-2018 7. Oliveira RC, Silva LF, Jesus MR, Santos TJ, Evaristo TN, Ribeiro WF, et al. O cuidado clínico e o processo de enfermagem em saúde mental: revisão integrativa da literatura. Rev Eletrôn Acervo Saúde. 2020;38:e2018. doi: http://doi.org/10.25248/reas.e2018.2020

8. Garcia TR. Classificação Internacional para a Prática de Enfermagem (CIPE ${ }^{\circledR}$ ): versão 2017. Porto Alegre: Artmed; 2018. $264 \mathrm{p}$

9. Carlson J. Consensus validation process: a standardized research method to identify and link the relevant NANDA, NIC, and NOC terms for local populations. Int J Nurs Terminol Classif. 2006;17(1):23-4. doi: http://doi. org/10.1111/j.1744-618X.2006.00020.x

10. Félix NDC, Nascimento MNR, Ramos NM, Oliveira $\mathrm{CJ}$, Nóbrega MML. Specialized nursing terminology for the care of people with metabolic syndrome. Esc Anna Nery. 2020;24(3):e20190345. doi: https://doi. org/10.1590/2177-9465-ean-2019-0345

11. Santana ET, Coutinho GG, Silva DVS, Bernardes TAA, Camisasca LR, Gusmão ROM, et al. Nursing diagnoses of NANDA-I taxonomy for the elderly in a long-term institution. Esc Anna Nery. 2021;25(1):e20200104. doi: https://doi. org/10.1590/2177-9465-ean-2020-0104

12. Risner PB. Diagnosis: analysis and synthesis of data.

In: Griffith-Kenney JW, Christensen PJ, editors. Nursing process application of theories, frameworks, and models. $2^{\mathrm{a}}$ ed. St. Louis: Mosby; 1986. p. 124-51

13. Christensen PJ, Kenney JW, editors. Nursing process: application of conceptual models. $4^{a}$ ed. St. Louis: Mosby; 1995

14. Carvalho EC, Dalm C, Herdman TH. Contribuição das linguagens padronizadas para a produção do conhecimento, raciocínio clínico e prática clínica da Enfermagem. Rev Bras Enferm. 2013;66(esp):134-41. doi: http://dx.doi. org/10.1590/S0034-71672013000700017

15. Borba LO, Maftum MA, Vayego AS, Kalinke LP, Ferreira ACZ, Capistrano FC. The mental disorder profile of patients treated at the center for psychosocial care (CAPS). Reme. 2017;21:e-1010. doi: http://doi. org/10.5935/1415-2762.20170020

16. Martins MAC, Hostalácio AM, Silva VLQ, Rosa WAG, Almeida DA. Perfil dos pacientes atendidos em um centro de atenção psicossocial, em Passos MG. Libertas. [Internet]. 2014 [Acesso 31 jul 2020];4(2):121-34. Disponível em: http://www.libertas.edu.br/revistas/index.php/ri clibertas/ article/view/59/55

17. Paiva RPN, Aguiar ASC, Cândido DA, Monteiro ARM, Almeida PC, Roscoche KGC, et al. Análise do perfil de usuários atendidos em um centro de atenção psicossocial.
J Health NPEPS. 2019;4(1):132-43. doi: http://doi. org/10.30681/252610103360

18. World Health Organization (SWI). Depression and Other Common Mental Disorders - Global Health Estimates [Internet]. Geneva: World Health Organization; 2017 [cited 2019 July 31]. 24 p. Available from: http://apps.who.int/ iris/bitstream/10665/254610/1/ WHO-MSD-MER-2017.2eng.pdf?ua $=1$

19. Boing AF, Melo GR, Boing AC, Moretti-Pires RO, Peres $K G$, Peres MA. Association between depression and chronic diseases: results from a population-based study. Rev Saúde Pública. 2012;46(4). doi: http://doi.org/10.1590/ S0034-89102012005000044

20. Magalhães LS, Andrade SMO. Depressão e Comportamento Suicida: Atenção Primária em Saúde. Rev Psicol Saúde. 2019;11(1):99-107. doi: http://doi. org/10.20435/pssa.v11i1.592

21. Jafelice GT, Marcolan JF. The multiprofessional work in the Psychosocial Care Centers of São Paulo State. Rev Bras Enferm. 2018;71(suppl 5):2259-66. doi: http://doi. org/10.1590/0034-7167-2017-0300

22. Nascimento LA, Leão A. Estigma social e estigma internalizado: a voz das pessoas com transtorno mental e os enfrentamentos necessários. Hist Cienc Saúde Manguinhos. 2019 [Acesso 7 set 2020];26(1):103-21. doi: https://doi. org/10.1590/s0104-59702019000100007

23. Santos SA, Carnut L. Trabalho, sentidos e saúde mental: percepção de participantes em um projeto para geração de renda. Semina: Ciênc Soc Hum. 2018 [Acesso 7 set 2020];39(2):159-80. doi: http://dx.doi. org/10.5433/1679-0383.2018v39n2p159

24. Cruz LS, Carmo DC, Sacramento DMS, Almeida MSP, Silveira HF, Ribeiro Júnior HL. Perfil de pacientes com transtornos mentais atendidos no Centro de Atenção Psicossocial do Município de Candeias - Bahia. Rev Bras Cienc Saúde. 2016;20(2)93-8. doi: http://doi.org/10.4034/ RBCS.2016.20.02.01

25. Rombaldi AJ, Silva MC, Gazalle FK, Azevedo MR, Hallal PC. Prevalência e fatores associados a sintomas depressivos em adultos do sul do Brasil: estudo transversal de base populacional. Rev Bras Epidemiol. 2010;13(4):620-9. doi: http://doi.org/10.1590/S1415-790X2010000400007

26. Stopa SR, Malta DC, Oliveira MM, Lopes CS, Menezes PR, Kinoshita RT. Prevalence of self-reported depression in Brazil: 2013 National Health Survey results. Rev Bras Epidemiol. 2015;18(Supl 2):170-80. doi: http://doi. org/10.1590/1980-5497201500060015

27. Hiany N, Vieira MA, Gusmão ROM, Barbosa SFA. Perfil Epidemiológico dos Transtornos Mentais na População Adulta no Brasil: uma revisão integrativa. Rev Enferm Atual. 2018 [Acesso 31 jul 2019];86. Disponível em: http://revistaenfermagematual.com/arquivos/ED_86_ REVISTA_24/42.pdf 
28. Gonçalves AMC, Teixeira MTB, Gama JRA, Lopes CS, Silva GA, Gamarra CJ, et al Prevalência de depressão e fatores associados em mulheres atendidas pela Estratégia de Saúde da Família. J Bras Psiquiatr. 2018;67(2):101-9. doi: http://doi.org/10.1590/0047-2085000000192

29. Conselho Federal de Enfermagem (BR). Resolução No 358 do Conselho Federal de Enfermagem, de 15 de outubro de 2009. Dispõe sobre a Sistematização da Assistência de Enfermagem e a implementação do Processo de Enfermagem em ambientes, públicos ou privados, em que ocorre o cuidado profissional de Enfermagem, e dá outras providências. [Internet]. Brasília; 2009 [Acesso 29 set 2018]. Disponível em: http://www.cofen.gov.br/resoluocofen-3582009_4384.html

30. Silva DVA, Sousa INM, Rodrigues CAO, Pereira FAF, Gusmão ROM, Araújo DD de. Nursing diagnoses in a homebased program: cross-mapping and NANDA-I Taxonom. Rev Bras Enferm. 2019;72(3):584-91. doi: http://doi. org/10.1590/0034-7167-2018-0323

31. Garcia TR, Cubas MR, organizadores. Diagnósticos, intervenções e resultados de enfermagem: subsídios para a sistematização da prática profissional. Rio de Janeiro: Elsevier; 2012

32. Antunes RJS, Manso FGCR. Diagnósticos de enfermagem num serviço de urgência psiquiátrica: contributos para a sistematização dos cuidados. Rev Enferm Ref. [Internet]. 2017;4(14):27-38. doi: http://doi.org/10.12707/RIV17040 33. Lopes JM, Fernandes SGG, Dantas FG, Medeiros JLA. Associação da depressão com as características sociodemográficas, qualidade do sono e hábitos de vida em idosos do Nordeste brasileiro: estudo seccional de base populacional. Rev Bras Geriatr Gerontol. 2015; 18(3):52131. doi: https://doi.org/10.1590/1809-9823.2015.14081 34. Garcia TR, Bartz CC, Coenen A. CIPE ${ }^{\circledR}$ : uma linguagem padronizada para a prática professional. In: Garcia TR, organizadora. Classificação Internacional para a Prática de Enfermagem - $\mathrm{CIPE}^{\circledR}$ : aplicação à realidade brasileira. Porto Alegre: Artmed; 2017

35. Gonçalves AMC, Teixeira MTB, Gama JRA, Lopes CS, Silva GA, Gamarra CJ, et al. Prevalência de depressão e fatores associados em mulheres atendidas pela Estratégia de Saúde da Família. J Bras Psiquiatr. 2018 [Acesso 31 jul 2020];67(2):101-9. doi: https://doi. org/10.1590/0047-2085000000192

36. Assumpção GLS, Oliveira LA, Souza MFS. Depressão e suicídio: uma correlação. Rev Pretextos. 2018 [Acesso 31 jul 2020];3(5):312-33. Disponível em: http://periodicos. pucminas.br/index.php/pretextos/article/view/15973
37. Cutcliffe JR. Research endeavours into suicide: a need to shift the emphasis. Br J Nurs. 2003;12(2):92-9. doi: http://doi.org/10.12968/bjon.2003.12.2.11058

38. Chachamovich E, Stefanello S, Botega N, Turecki G. Which are the recent clinical findings regarding the association between depression and suicide? Rev Bras Psiquiatr. 2009;31(Supl I):S18-25. doi: http://doi. org/10.1590/S1516-44462009000500004

39. Fernandes FY, Freitas BHBM, Marcon SR, Arruda VL, Lima NVP, Bortolini J, et al. Suicide trend among Brazilian adolescents between 1997 and 2016. Epidemiol Serv Saúde. 2020;29(4):e2020117. doi: https://doi.org/10.1590/ SciELOPreprints. 964

40. Ministério da Saúde (BR). Secretaria de Atenção à Saúde. Departamento de Ações Programáticas Estratégicas, Área Técnica de Saúde Mental. Prevenção do Suicídio: manual dirigido a profissionais das equipes de saúde mental. [Internet]. Ministério da Saúde, Organização Pan-Americana da Saúde; 2006 [Acesso 31 jul 2019]. 76 p. Disponível em: http://bvsms.saude.gov.br/bvs/publicacoes/manual_ editoracao.pdf

\section{Contribuição dos autores}

Concepção e desenho da pesquisa: Ricardo Otávio Maia Gusmão, Diego Dias de Araújo. Obtenção de dados: Natália Hiany Fonseca Santos, Daniel Vinícius Alves Silva, Deborah Fernanda Nunes Moreira. Análise e interpretação dos dados: Ricardo Otávio Maia Gusmão, Natália Hiany Fonseca Santos, Daniel Vinícius Alves Silva, Deborah Fernanda Nunes Moreira, Maria Aparecida Vieira, Diego Dias de Araújo. Análise estatística: Ricardo Otávio Maia Gusmão, Maria Aparecida Vieira, Diego Dias de Araújo. Redação do manuscrito: Ricardo Otávio Maia Gusmão, Natália Hiany Fonseca Santos, Daniel Vinícius Alves Silva, Deborah Fernanda Nunes Moreira, Maria Aparecida Vieira, Diego Dias de Araújo. Revisão crítica do manuscrito quanto ao conteúdo intelectual importante: Ricardo Otávio Maia Gusmão, Natália Hiany Fonseca Santos, Daniel Vinícius Alves Silva, Deborah Fernanda Nunes Moreira, Maria Aparecida Vieira, Diego Dias de Araújo.

Todos os autores aprovaram a versão final do texto. Conflito de interesse: os autores declararam que não há conflito de interesse.

Recebido: 03.07.2020 Aceito: 13.09 .2020 